\title{
Dependence of the ray transference of model eyes on the frequency of light
}

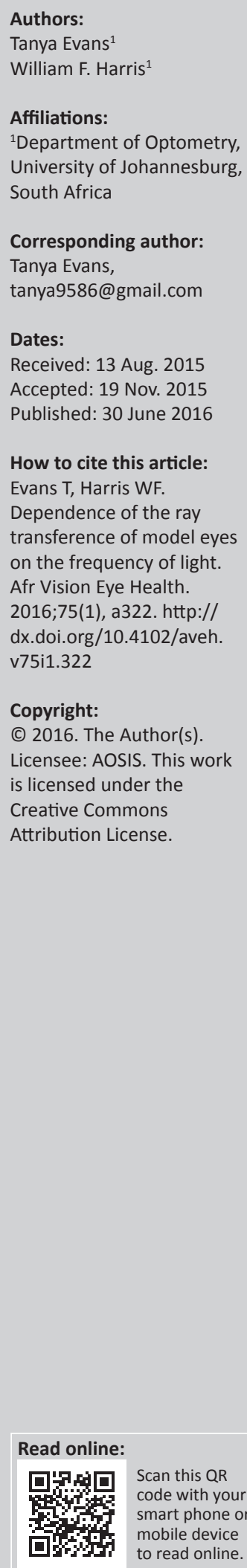

The transference defines the first-order character of an optical system; almost all the system's optical properties can be calculated from it. It is useful, therefore, to have some idea of how it depends on the frequency of light. We examine the dependence for two Gaussian eyes. It turns out to be nearly linear for all four fundamental properties. The result is an equation for the dependence of the transference on frequency which is almost symplectic. We also transform the transference into Hamiltonian space, obtain equations for the least-squares straight line for the three independent transformed properties and map them back to the group of transferences. The result is an equation for the dependence of the transference on frequency which is exactly symplectic and therefore representative of an optical system. The results may approximate those of real eyes and give estimates of the dependence of almost all optical properties on frequency.

\section{Introduction}

The ray transference is of central importance in linear optics. Nearly all the familiar optical properties of an eye such as power, refractive compensation, magnification, and cardinal points can be derived from the transference. It is therefore useful to have some idea of how transference depends on the frequency of light. In this presentation, we examine the dependence of the transference of the reduced eye $\mathrm{e}^{1,2}$ and Le Grand's four-surface schematic eye $\mathrm{e}^{3}$ on frequency with the objective of obtaining an equation for the dependence. Consequently, the dependence of the eye's optical properties on frequency, as well as their chromatic difference between two frequencies, can be obtained from the frequency-dependent transference. ${ }^{2}$ This forms part of a much larger study. Many of these chromatic properties can be generalised to astigmatic heterocentric eyes.

\section{Method}

The underlying method used here is that of first-order optics. We make use of the ray transference, which is a complete representation of the first-order effects of an optical system on the rays traversing it. ${ }^{4}$ We represent the transference as

$\mathbf{S}=\left(\begin{array}{ll}A & B \\ C & D\end{array}\right)$

where $A$ the dilation, $B$ the disjugacy, $C$ the divergence, and $D$ the divarication are the four fundamental properties of the Gaussian system. ${ }^{5}$

Being a member of the symplectic group, the transference has unit determinant. ${ }^{5,6}$ Symplectic matrices are closed under multiplication, inversion, and transposition but are not closed under addition nor multiplication by a scalar. ${ }^{5,6}$ This creates problems when doing quantitative analyses on sets of transferences. ${ }^{6}$ To overcome this limitation, we make use of the mapping from the symplectic group to the set of Hamiltonian matrices. The set of all Hamiltonian matrices defines a linear (vector) space and is, therefore, closed under matrix addition and multiplication by a scalar. ${ }^{5,6}$ This makes the set of Hamiltonian matrices suitable for quantitative analysis including conventional statistical analysis. We explore two mappings. First, the principal matrix logarithm of a symplectic matrix is a Hamiltonian matrix and inversely the matrix exponential of a Hamiltonian matrix is a symplectic matrix. Second, the Cayley transform, being its own functional

Note: This work was presented as a poster presentation at Visual and Physiological Optics 2014 conference in Wrocław, Poland. 
inverse, provides a mapping between symplectic and Hamiltonian matrices. The Cayley transform is defined as ${ }^{7}$

$$
\hat{\mathbf{S}}=(\mathbf{I}-\mathbf{S})(\mathbf{I}+\mathbf{S})^{-1}
$$

where the caret $\left({ }^{\wedge}\right)$ denotes the Hamiltonian transformed transference.

We are interested in the dependence of the transference on the frequency of light across the visible spectrum, $430 \mathrm{THz}$ to $750 \mathrm{THz}\left(\times 10^{12} \mathrm{~s}^{-1}\right)$. Frequency is independent of the medium, whereas wavelength is not and energy is proportional to frequency, both good reasons for studying the dependence of properties on the frequency of light rather than on wavelength. ${ }^{8}$ When obtaining the transference, it is the refractive index that is dependent on frequency. We make use of the formula for the refractive index as a function of wavelength developed for the chromatic eye $e^{9}$ and the formulae for the refractive indices as functions of wavelength for the cornea, aqueous, lens, and vitreous developed by Villegas et al. ${ }^{10}$ based on the polynomial fit of Le Grand's findings ${ }^{11}$ for the four-surface schematic eye. Both sets of equations are based on experimental findings. The refractive index of air is approximated by $n_{0}=1$. The transferences were calculated as described elsewhere. ${ }^{2,12}$

\section{Results}

The dependence of the transference on the frequency of light is shown (Figure 1) for the reduced eye (blue) and Le Grand's eye (black) and is very nearly linear for each of the fundamental properties. The dashed straight lines shown in the figure are obtained using the least-squares method. The formula for the straight lines as a function of frequency $(v)$ is given by

$\mathbf{S}=\left(\begin{array}{ll}a_{1} & b_{1} \\ c_{1} & d_{1}\end{array}\right) v+\left(\begin{array}{ll}a_{2} & b_{2} \\ c_{2} & d_{2}\end{array}\right)$

with constants given in Box 1 for the reduced and Le Grand's eyes. This gives us a good approximation for the transference of a Gaussian eye as a function of frequency.

Transforming the frequency-dependent transferences into Hamiltonian matrices, we are able to obtain the least-squares straight line for each of the three independent entries in Hamiltonian space, which we map back to symplectic matrices. This allows us to obtain an expression for the dependence of the transference on the frequency of light,
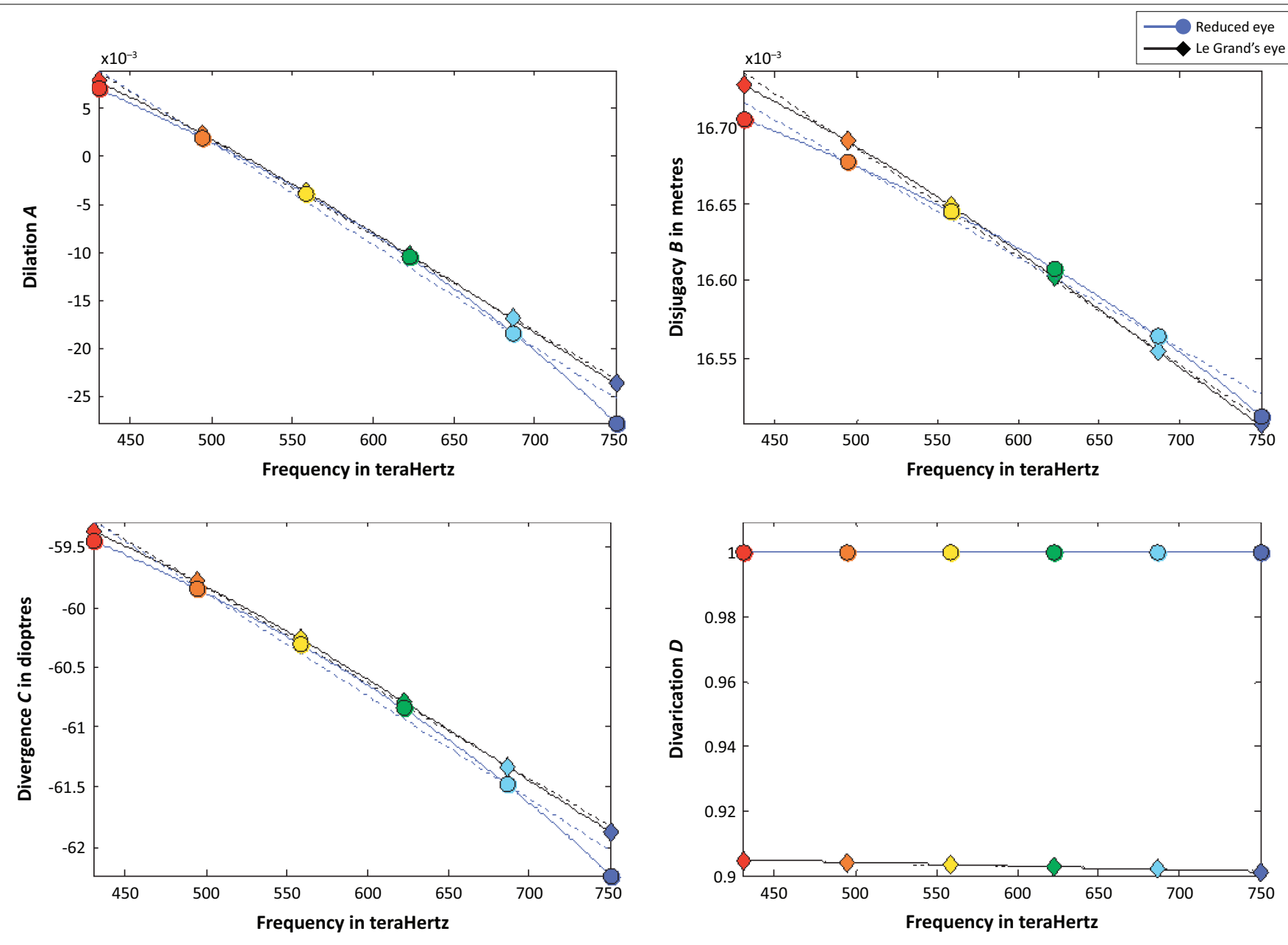

FIGURE 1: The fundamental properties of the reduced and Le Grand's eyes as functions of frequency. The least-squares straight lines (Equation 3) are shown with dashed

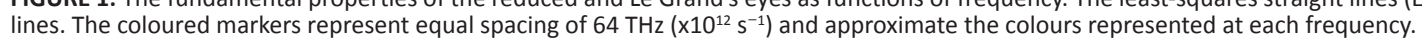


BOX 1: Constants for Equation 3, the units being picoseconds $\left(\times 10^{-12} \mathrm{~s}\right)$, metres and dioptres $\left(\mathrm{m}^{-1}\right)$.

\begin{tabular}{|llll}
\hline & Reduced eye & & \multicolumn{2}{c}{ Le Grand's eye } \\
$a_{1}=-1.069 \times 10^{-4} \mathrm{ps}$ & $a_{2}=0.0549$ & $a_{1}=-0.997 \times 10^{-4} \mathrm{ps}$ & $a_{2}=0.0516$ \\
$b_{1}=-5.939 \times 10^{-7} \mathrm{mps}$ & $b_{2}=16.972 \times 10^{-3} \mathrm{~m}$ & $b_{1}=-7.034 \times 10^{-7} \mathrm{mps}$ & $b_{2}=17.038 \times 10^{-3} \mathrm{~m}$ \\
$c_{1}=-8.605 \times 10^{-3} \mathrm{Dps}$ & $c_{2}=-55.579 \mathrm{D}$ & $c_{1}=-7.975 \times 10^{-3} \mathrm{Dps}$ & $c_{2}=-55.849 \mathrm{D}$ \\
$d_{1}=0 \mathrm{ps}$ & $d_{2}=1$ & $d_{1}=-1.115 \times 10^{-5} \mathrm{ps}$ & $d_{2}=0.910$ \\
\hline
\end{tabular}

BOX 2: Constants for Equation 4 for the Cayley transform.

\begin{tabular}{|llll}
\hline & Reduced eye & & \multicolumn{2}{l}{ Le Grand's eye } \\
$\hat{a}_{1}=4.780 \times 10^{-5} \mathrm{ps}$ & $\hat{a}_{2}=0.309$ & $\hat{a}_{1}=4.263 \times 10^{-5} \mathrm{ps}$ & $\hat{a}_{2}=0.289$ \\
$\hat{b}_{1}=0 \mathrm{mps}$ & $\hat{b}_{2}=-11.111 \times 10^{-3} \mathrm{~m}$ & $\hat{b}_{1}=4.633 \times 10^{-8} \mathrm{mps}$ & $\hat{b}_{2}=-11.509 \times 10^{-3} \mathrm{~m}$ \\
$\hat{c}_{1}=7.206 \times 10^{-3} \mathrm{Dps}$ & $\hat{c}_{2}=36.298 \mathrm{D}$ & $\hat{c}_{1}=7.111 \times 10^{-3} \mathrm{Dps}$ & $\hat{c}_{2}=37.630 \mathrm{D}$ \\
\hline
\end{tabular}

BOX 3: Constants for Equation 4 for the logarithmic transform.

\begin{tabular}{|llll}
\hline & Reduced eye & & \multicolumn{1}{c|}{ Le Grand's eye } \\
$\hat{a}_{1}=-7.905 \times 10^{-5} \mathrm{ps}$ & $\hat{a}_{2}=-0.564$ & $\hat{a}_{1}=-6.887 \times 10^{-5} \mathrm{ps}$ & $\hat{a}_{2}=-0.523$ \\
$\hat{b}_{1}=-2.491 \times 10^{-7} \mathrm{~m}$ ps & $\hat{b}_{2}=20.281 \times 10^{-3} \mathrm{~m} \mathrm{ps}$ & $\hat{b}_{1}=-3.567 \times 10^{-7} \mathrm{~m} \mathrm{ps}$ & $\hat{b}_{2}=20.781 \times 10^{-3} \mathrm{~m}$ \\
$\hat{c}_{1}=-12.145 \times 10^{-3} \mathrm{D} \mathrm{ps}$ & $\hat{c}_{2}=-66.312 \mathrm{D}$ & $\hat{c}_{1}=-11.743 \times 10^{-3} \mathrm{Dps}$ & $\hat{c}_{2}=-68.009 \mathrm{D}$ \\
\hline
\end{tabular}

which is exactly symplectic. The least-squares straight line in Hamiltonian space is

$\hat{\mathbf{S}}=\left(\begin{array}{cc}\hat{a}_{1} & \hat{b}_{1} \\ \hat{c}_{1} & -\hat{a}_{1}\end{array}\right) v+\left(\begin{array}{cc}\hat{a}_{2} & \hat{b}_{2} \\ \hat{c}_{2} & -\hat{a}_{2}\end{array}\right)$

with the constants for the reduced and Le Grand's eyes given in Box 2 for the Cayley transform and Box 3 for the logarithmic transform. To obtain the transference as a function of frequency, one needs to map the transformed transference back to its respective transference using either the Cayley transform (Equation 2) or the matrix exponential. The frequency-dependent transference is easy to obtain using pencil and paper and either the Cayley transform of Equation 2 or, easier still

$\mathbf{S}=\frac{2(\mathbf{I}-\hat{\mathbf{S}})}{1+\operatorname{det} \hat{\mathbf{S}}}-\mathbf{I}$

[Eqn 5]

On the other hand, the logarithmic transform requires the use of sophisticated matrix software.

\section{Conclusion}

The four fundamental properties of a Gaussian eye are shown to have a very nearly linear dependence on frequency. An equation is obtained for the least-squares straight line dependence of the fundamental properties on frequency, the estimated transference is almost symplectic. A transference, that is, a matrix which is exactly symplectic, is obtained by fitting a straight line in Hamiltonian space, giving the dependence of the transference of a Gaussian eye on the frequency of light across the visible spectrum. These equations allow one to write approximate equations for the dependence of almost all the optical properties of the eye, both fundamental and derived, on frequency.

\section{Acknowledgements}

W.F.H. gratefully acknowledges support from the National Research Foundation of South Africa.

\section{Competing interests}

The authors declare that they have no financial or personal relationships which may have inappropriately influenced them in writing this article.

\section{Authors' contributions}

This work is based on research by T.E. towards a higher degree under the guidance of W.F.H.

\section{References}

1. Emsley HH. Visual optics. 4th ed. London: Hatton Press Ltd; 1950; p. 523-544.

2. Evans T, Harris WF. Dependence of a reduced eye on frequency of light. S Afr Optom. 2011;70:149-155. http://www.saoptometrist.co.za/EVANS_DEC2011.pdf

3. Le Grand Y. Optique Physiologique. Tome Premier, le Dioptrique de I'CEil et sa Correction. Paris: Revue d'Optique; 1945; p. 50-51.

4. Torre A. Linear ray and wave optics in phase space. Amsterdam: Elsevier; 2005; p. 60.

5. Harris WF. The log-transference and an average Gaussian eye. S Afr Optom. 2005;64:84-88

6. Harris WF. Symplecticity and relationships among the fundamental properties in linear optics. S Afr Optom. 2010;69:3-13.

7. Bernstein DS. Matrix mathematics. Theory, facts, and formulas. 2nd ed. Princeton, NJ: Princeton University Press; 2009; p. 208-209, 238-239.

8. Pease PL, Barbeito R. Axial chromatic aberration of the human eye: Frequency or wavelength? Ophthalmic Physiol Opt. 1989;9:215-217. http://dx.doi.org/10.1111/ j.1475-1313.1989.tb00845.x

9. Thibos LN, Ye M, Zhang X, Bradley A. The chromatic eye: A new reduced-eye model of ocular chromatic aberration in humans. Appl Opt. 1992;31:3594-3600. $\mathrm{http}: / / \mathrm{dx}$.doi.org/10.1364/AO.31.003594

10. Villegas ER, Carretero L, Fimia A. Le Grand eye for the study of ocular chromatic aberration. Ophthalmic Physiol Opt. 1996;16:528-531. http://dx.doi.org/10.1016/ 0275-5408(96)00007-5

11. Le Grand Y. Optique Physiologique. Tome Troisième, l'Espace Visuel. Paris: Revue d'Optique; 1956; p. 9-27.

12. Guillemin V, Sternberg S. Symplectic techniques in physics. Cambridge: Cambridge University Press; 1984; p. 9-27. 